\title{
Alarm Pheromone Composition and Behavioral Activity in Fungus-Growing Ants
}

\author{
Victoria C. Norman ${ }^{1} \cdot$ Thomas Butterfield $^{1} \cdot$ Falko Drijfhout ${ }^{2} \cdot$ Kiah Tasman $^{1}$ • \\ William O. H. Hughes ${ }^{1}$
}

Received: 18 October 2016 /Revised: 6 January 2017 / Accepted: 8 February 2017 / Published online: 28 February 2017

(C) The Author(s) 2017. This article is published with open access at Springerlink.com

\begin{abstract}
Chemical communication is a dominant method of communication throughout the animal kingdom and can be especially important in group-living animals in which communicating threats, either from predation or other dangers, can have large impacts on group survival. Social insects, in particular, have evolved a number of pheromonal compounds specifically to signal alarm. There is predicted to be little selection for interspecific variation in alarm cues because individuals may benefit from recognizing interspecific as well as conspecific cues and, consequently, alarm cues are not normally thought to be used for species or nestmate recognition. Here, we examine the composition of the alarm pheromones of seven species of fungusgrowing ants (Attini), including both basal and derived species and examine the behavioral responses to alarm pheromone of Acromyrmex leaf-cutting ants, the sister genus to the highly studied Atta leaf-cutting ants. We find surprisingly high interspecific variation in alarm pheromone composition across the attine phylogeny. Interestingly, the active component of the alarm pheromone was different between the two leaf-cutting ant genera. Furthermore, in contrast to previous studies on Atta, we found no differences among morphological castes in their responses to alarm pheromone in Acromyrmex but we did find differences in responses among putative age classes. The results suggest that
\end{abstract}

Electronic supplementary material The online version of this article (doi:10.1007/s10886-017-0821-4) contains supplementary material, which is available to authorized users.

Victoria C. Norman

V.Norman@sussex.ac.uk

1 School of Life Sciences, University of Sussex, Brighton, East Sussex BN1 9QG, UK

2 Chemical Sciences Research Centre, Keele University, Staffordshire, UK the evolution of alarm communication and signaling within social insect clades can be unexpectedly complex and that further work is warranted to understand whether the evolution of different alarm pheromone compounds is adaptive.

Keywords Leaf-cutting ant · Mandibular gland · Caste · Attini $\cdot$ Division of labor $\cdot$ Alarm pheromone

\section{Introduction}

Alarm communication is shown by a wide variety of organisms, including vertebrates, invertebrates and even plants (Chivers and Smith 1998; Shah 2009; Wyatt 2014). Alarm communication is particularly beneficial when individuals live in groups, because the rapid communication of threats to group members enables groups to form collective responses to stimuli (Nault and Phelan 1984; Verheggen et al. 2010). In many organisms, these alarm signals are visual (Murphy 2006; le Roux et al. 2008), but in insects they are predominantly chemical, with alarm pheromones being the secondly most commonly produced class of compounds among this group of organisms (Regnier and Law 1968; Verheggen et al. 2010).

Alarm communication is particularly diverse in the eusocial insects such as ants, in which a wide variety of compounds function as stimuli for alarm behaviors (Bortolotti and Costa 2014; Cammaerts et al. 1983; Crewe et al. 1972). These behaviors allow individuals within a colony to respond rapidly and appropriately to an alarm stimulus. There are two main behavioral responses to alarm cues, each of which serve separate functions (Verheggen et al. 2010; Wilson and Regnier 1971). The first is a panic response, in which responders show escape or flight behaviors to disperse from the threat. The second is an aggressive response, in which workers are attracted to, and attack, the threat. These responses can depend 
upon a variety of factors, including concentration of pheromone, compounds within the pheromone, colony size, and the spatial context of the communication (Hölldobler and Wilson 1990; Hughes and Goulson 2001; Kerr et al. 1973; Vander Meer and Alonso 1998).

We may also expect differences among individual workers in their response to alarm cues. Polyethism in complex eusocial insect societies can be based on age (temporal polyethism) or morphological phenotype (caste). Many insects show a specialized defensive caste, be it guards in honey bee colonies, or morphologically specialized soldiers in stingless bee, ant, termite, aphid or thrips colonies (Grüter et al. 2012; Hölldobler and Wilson 2010a; Shibao 1998). These individuals are often the first line of defence for the group and respond more aggressively to defensive stimuli (Whitehouse and Jaffe 1996). There is variation among colonies in alarm responses as well as between species; for example, as in the greater defensiveness of Africanized honey bees compared to their European counterparts (Collins et al. 1982; Giray et al. 2000). However, the reduced need for specificity in alarm pheromones compared to other pheromones means that there is predicted to be relatively little selection for the evolution of species-specific alarm pheromones (Blum 1969; Regnier and Law 1968; Vander Meer and Alonso 1998). Indeed, in other organisms, such as fish or crustaceans, a lack of species specificity can be an advantage in alarm cues (Commens and Mathis 1999; Laforsch et al. 2006).

The tribe Attini provides an ideal system for testing this hypothesis within social insects. This clade exhibits varying levels of social complexity. Leaf-cutting ants (Atta and Acromyrmex) are the most derived clade within the fungusgrowing ants (tribe Attini), all of which are characterized by culturing a mutualistic fungal crop. The two ecologically dominant leaf-cutting ant genera are distinguished from other attines by cultivating their fungal crop, generally, on fresh vegetation and having much larger and more complex societies, with thousands to millions of polymorphic workers compared to tens to hundreds of monomorphic workers in the basal attines (Weber 1972). The higher leaf-cutting genera of Acromyrmex and Atta show some of the most complex forms of division of labor with extreme caste polymorphism, in which workers can vary in size by as much as six-fold ( Hölldobler and Wilson 2010b; Wilson 1980). Within leafcutting ant colonies, smaller workers tend to carry out internal work within the nest, such as caring for the brood and fungus garden, larger workers tend to carry out foraging and nest defence, while soldiers (only present in Atta) specialize normally in nest defence (Wetterer 1995; Wilson 1980). As with many other ant and bee species, the alarm pheromone in leafcutting ants is produced by the mandibular glands and is released when the mandibles are gaped in response to an alarm stimulus (Blum 1969; Verheggen et al. 2010). The compositions of the alarm pheromones in Atta have been relatively well studied, and all six of the species so far investigated (A. bisphaerica, A. capiguara, A. laevigata, A. opaciceps and $A$. sexdens) have 4-methyl-3-heptanone as the predominant and most active component (Blum et al. 1968; Crewe and Blum 1972; Do Nascimento et al. 1993; Francelino et al. 2006; Hernandez et al. 1999; Hughes et al. 2001a; Moser et al. 1968;). Depending on the species and caste, the alarm pheromone of Atta can also contain other volatile compounds, with as many as 41 volatile compounds found in head extracts (Hughes et al. 2001a), but the roles of these other compounds are unclear. There is also clear evidence of polymorphism in the composition, activity and receptiveness of Atta ants to alarm pheromone. While large workers and particularly soldiers tend to produce relatively complex pheromonal mixtures, the alarm pheromone of small workers (minims) in Atta is simpler, consisting predominantly of 4-methyl-3heptanone (Do Nascimento et al. 1993; Hernandez et al. 1999; Hughes et al. 2001a). This suggests that the small workers in Atta may produce a particularly potent alarm pheromone in terms of composition, as the quantities they produce are lower, due to their smaller mandibular glands, than those of their larger nestmates (Hernández and Caetano 1995). Although all castes respond to alarm pheromone (Wilson 1980), field studies have found that smaller workers, although less abundant on foraging trails, are disproportionately abundant in the ants responding to alarm stimuli, suggesting that they may play a key role in detecting and responding to threats (Hughes and Goulson 2001).

The rich literature on the composition and behavioral activity of alarm pheromones in Atta leaf-cutting ants contrasts markedly with the very limited investigation of alarm pheromones in other fungus-growing ants. Only two studies have investigated the chemical composition of the mandibular gland pheromones in other Attini, and these suggest intergeneric differences, with eight Trachymyrmex species appearing to lack 4methyl-3-heptanone (Adams et al. 2012; Crewe and Blum 1972). These results are intriguing because they suggest that different fungus-growing ant taxa may have evolved different alarm pheromone compounds, in contrast to the predicted lack of selection for interspecific variation in alarm pheromone. However, detailed analyses of the composition of alarm pheromones, and controlled assays of behavioral activity, are needed for Acromyrmex and other attines in order to resolve this. Here, we identify and compare the chemical composition of the alarm pheromones of two species of Atta that have not previously been investigated (At. colombica and At. cephalotes), two species of Acromyrmex (Ac. echinatior and Ac. octospinosus), two other 'higher' attines (Sericomyrmex amabilis and Trachymyrmex cornetzi), and the 'lower' attine Apterostigma pilosum (a coral fungus ant, representing the most basal of the attines). We also examined the behavioral activity elicited by the most abundant components of the alarm pheromones for the two Acromyrmex species, and compared this with the 
behavioral responses of the two Atta species to their most behaviorally active components. Finally, we examined how caste and age of Acromyrmex workers affect their responsiveness to alarm pheromone. We did this using a precise, individual-level assay based on the mandible opening response (Guerrieri and D'Ettorre 2008), a response which is stimulated in ants by alarm pheromones regardless of whether ants exhibit a panic or aggressive response (Hölldobler and Wilson 1990; Hughes et al. 2001b; Norman et al. 2014).

\section{Methods and Materials}

Chemical Composition of Alarm Pheromone in Attine Ants Workers were collected from colonies of Ap. pilosum, S. amabilis, T. cornetzi, Ac. echinatior, Ac. octospinosus, At. cephalotes and At. colombica that had been collected from Gamboa, Panama in May 2013 and maintained in the laboratory for 6-18 months. We collected 20 workers from each of 2-4 colonies of the monomorphic Ap. pilosum (3 colonies), T. cornetzi ( 2 colonies) and $S$. amabilis (4 colonies). For the polymorphic leaf-cutting ants, we collected 20 small $(<1.5 \mathrm{~mm} \mathrm{~mm}$ head width) and 20 large $(2.0-3.0 \mathrm{~mm}$ head width) workers from 2 to 4 colonies of Ac. echinatior (4 colonies), Ac. octospinosus (3 colonies), At. colombica (2 colonies) and At. cephalotes ( 2 colonies). Ants were immediately cooled on ice after collection, and their heads removed and placed into $100 \mu \mathrm{l}$ hexane (97\% pure) containing $10 \mathrm{ng} / \mathrm{ant}$ head of an internal standard (5-methyl-3-heptanone 97\%, Sigma-Aldrich, St Louis, MO). Heads were crushed thoroughly with a clean glass rod, vortexed for $60 \mathrm{~s}$, placed in a sonic bath for $60 \mathrm{~s}$, spun down for $60 \mathrm{~s}$, filtered through a glass Pasteur pipette with a plug of clean glass wool, and the filtered extract stored at $-20{ }^{\circ} \mathrm{C}$ until analysis. Crushed heads were used because previous work showed no difference in the composition of major volatiles present in mandibular glands and that released by crushed heads (Blum et al. 1968; Do Nascimento et al. 1993; Francelino et al. 2008; Hernández et al. 2002; Moser et al. 1968; Murakami et al. 2000; VN, FD and WOHH, unpubl. Data)

The sample extracts were analyzed by gas chromatography/mass spectrometry (GC/MS), using a Perkin Elmer Autosystem XL GC and TurboMass MS. The column used was a Supelco SLB-5 ms, $30 \mathrm{~m} \times 0.25 \mathrm{~mm}$ ID $\times$ $0.25 \mu \mathrm{m}$ film thickness (Sigma-Aldrich). The temperature program was $40{ }^{\circ} \mathrm{C}$ (held for $3 \mathrm{~min}$ ), then $10{ }^{\circ} \mathrm{C} \cdot \mathrm{min}^{-1}$ to $75^{\circ} \mathrm{C}, 20{ }^{\circ} \mathrm{C} \cdot \mathrm{min}^{-1}$ to $300{ }^{\circ} \mathrm{C}$, and held for $5 \mathrm{~min}$. Samples were injected using a splitless injector with a purge time of $1.0 \mathrm{~min}$; the inlet temperature was $250{ }^{\circ} \mathrm{C}$ and injection volume $0.3 \mu \mathrm{l}$. The carrier gas was helium at $1.3 \mathrm{ml} . \mathrm{min}^{-1}$. The instrument was operated in full scan mode with range $m / z 20$ 650. Transfer line and MS source temperatures were $300{ }^{\circ} \mathrm{C}$ and $250{ }^{\circ} \mathrm{C}$, respectively. Compounds were identified by searching a database (NIST MSSearch $2.0 \mathrm{~g}$ ) for matching mass spectra then confirmed by comparing retention times and mass spectra with authentic standards.

Experiment 1: Behavioral Activity of Compounds in LeafCutting Ants In order to determine the behaviorally active components of the alarm pheromone, the mandible-opening response (MOR) assay was used (Guerrieri and D'Ettorre 2008; Norman et al. 2014). This individual-level assay is ideal because individual ants can be isolated and their threat response toward specific chemical stimuli under controlled conditions can be easily ascertained by whether or not the focal ant gapes its mandibles after exposure to the stimulus (indicating recognition of a threat, and therefore capturing both panic and aggression responses) (Hölldobler and Wilson 1990; Hughes et al. 2001b; Norman et al. 2014).

To carry out the MOR assay, ants were chilled on ice and then harnessed in $0.2 \mathrm{ml}$ pipette tips (Starlab, Bucks, UK), cut at the apex and through which the head of the ant was passed, and secured with a thin strip of masking tape. Ants were left for $2 \mathrm{~h}$ in the harness to acclimatize before being assayed, and for at least $30 \mathrm{~min}$ between chemical stimuli to avoid habituation. The order of presentation of the stimuli to individuals was randomized. In order to test which of the chemicals identified in the alarm pheromone extract were active, compounds identified as consistently present in the extracts of either Atta or Acromymex species were tested on their respective species using the MOR. Neat compounds were used for this assay, as preliminary data showed these elicited the highest levels of response (V. C. N., unpublished data). We compared the responses of 30 large workers (2.0-2.5 mm head width) and 30 small workers (1.0-1.5 mm head width) for each species, sampled equally from five colonies of Ac. echinatior, Ac. octospinosus and At. colombica, or from the single available colony of At. cephalotes. To simulate the volatile emission of alarm pheromone by ants, a $20 \mu \mathrm{l}$ drop of neat compound was placed on filter paper $(10 \times 20 \mathrm{~mm}) 10 \mathrm{~mm}$ from the antennae of the focal ant, and the response of the ant recorded. A $20 \mu \mathrm{l}$ drop of water applied in the same way was used as a negative control, and the crushed head of a freshly freeze-killed nestmate used as a positive control.

Experiment 2: the Effect of Caste and Age on Alarm Response in Acromyrmex To determine if the caste or age of Ac. echinatior and Ac. octospinsus leaf-cutting ant workers affected their response to alarm pheromone, we again utilized the MOR assay. To examine the effect of caste, we compared the responses of 30 small workers, 30 medium workers, and 30 large workers $(1.25 \pm 0.03,1.65 \pm 0.02$, and $2.09 \pm 0.03 \mathrm{~mm}$ head widths, respectively, sampled equally from five colonies of each species). All ants were of medium cuticular coloration, indicating similar age (Armitage and Boomsma 2010). This was confirmed by quantifying the 
cuticular coloration of a subset of 30 ants/caste from a dorsal photograph taken with a Canon EOS 350d dSLR camera and Canon EF $100 \mathrm{~mm} \mathrm{f} / 2.8$ Macro lens under constant lighting. Images were imported into ImageJ software and converted to grayscale, giving a reading of 0 (pure black) to 256 (pure white). Cuticular color was quantified using the mean value of the middle third of the femur of one of the rear legs, as in Armitage and Boomsma (2010). To examine the effect of age on the threat response, ants of similar size $(1.2-1.8-\mathrm{mm}$ head width) and of three different putative age classes (young, medium and old) were selected based on their cuticular coloration. Six ants of each age class were chosen from each of the five colonies for each species, giving 90 ants in total per species. To confirm age class, each ant was photographed and cuticular coloration quantified as described above. Mean \pm s.e. color for each of the three putative age categories was: young $93.4 \pm 1.4$, medium $70.5 \pm 0.75$ and old $57.6 \pm 0.93$ (Fig. S2). In both the caste and age experiments, we tested the responses of ants to the most behaviorally active compound (3-octanol for Ac. echinatior and 3-octanone for Ac. octospinosus) at a dosage corresponding to the amount found in a single large worker ant head (25 $\mathrm{ng}$ for $A c$. echinatior, $135 \mathrm{ng}$ for Ac. octospinosus). Compounds were dissolved in hexane to $1 \mathrm{ng} / \mu \mathrm{l}$, placed on filter paper, left for $15 \mathrm{~s}$ to allow the solvent to evaporate, and the filter paper $(10 \times 20 \mathrm{~mm})$ placed within $10 \mathrm{~mm}$ of the antennae of the focal ant. Worker's responses to these 'head-realistic' doses were compared to a solvent negative control (prepared in the same way as the head-realistic doses, but with an equivalent volume of hexane only), and a crushed head of a freshly freeze-killed nestmate as a positive control.

Experiment 3: Colony-Level Assay To confirm that the MOR assay was appropriate for detecting alarm responses, we also carried out colony-level assays using Ac. echinatior and Ac. octospinosus to confirm, in a more realistic colony set-up, that the ants exhibited alarm behaviors in response to the compounds and that individuals showed mandible gaping when alarmed. For each of the compounds found consistently in the extracts of each species, $50 \mathrm{~mm}$ filter paper discs containing the equivalent amount found in one crushed ant's head were used as alarm stimuli. Once applied to the disc, the solvent was left to evaporate for $15 \mathrm{~s}$ before the disc was placed at least $50 \mathrm{~mm}$ away from the nest entrance. Each assay was filmed for $1 \mathrm{~min}$ with snapshot behaviors recorded at $10 \mathrm{~s}$ intervals during this period. The number of ants gaping the mandibles was recorded at each $10 \mathrm{~s}$, as well as the number of ants present on the filter paper to quantify attraction or arrestment. This was carried out for four Ac. octospinosus colonies and three Ac. echinatior colonies. A crushed head of a freshly freeze-killed nestmate was used as a positive control.
Experiment 4: Confirmation of Behavioral Responses to Volatiles Released by Ants Finally, to confirm that focal ants were reacting to volatiles released by alarmed ants, and not just to other compounds in crushed whole heads, we carried out a further experiment utilizing the MOR assay. Focal ants were exposed to five stimuli in random order: 1) a live alarmed nestmate (with gaping mandibles indicating that the stimulus ant was alarmed and releasing alarm pheromone) held $10 \mathrm{~mm}$ away from the head of the focal ant, 2) a dead nestmate, which could not be releasing alarm pheromone, as a control, 3) eight live nestmates, in a sealed pot ( $25 \mathrm{~mm}$ diam., $40 \mathrm{~mm}$ height), that had been alarmed for $20 \mathrm{~s}$, with the pot then placed $10 \mathrm{~mm}$ from the focal ant and opened, 4) the same treatment but under red light, to ensure focal ants were not reacting to the sight of alarmed ants, and 5) an empty pot as a control. These assays were carried out for 40 Ac. echinatior individuals (sampled evenly from four colonies) and $50 \mathrm{Ac}$. octospinosus individuals (sampled evenly from five colonies).

Statistical Analysis The programme PRIMER 6, version 6.1.13, was used with the permutational multivariate analysis of variance (PERMANOVA) version 1.0.3 for multivariate analysis of percentage composition to determine differences among species of the compounds present in the alarm pheromone. This analysis, a non-parametric MANOVA, has the advantage of being free from assumptions on data distribution (Anderson et al. 2008). All multivariate analyses were carried out using 9999 permutations on a resemblance matrix using Euclidian distance estimates. We used a one-factor PERMANOVA design, with species identity as a fixed factor. All peaks identified as present in the alarm pheromone (41 in total over the 7 species) were included in the analysis. A further PERMANOVA model was constructed to calculate posthoc pairwise comparisons between species groups. We also carried out a one-way similarity of percentage analysis (SIMPER), to analyze further qualitative differences among species in alarm pheromone composition. This calculates the contributions of specific chemicals to the separation of species by the chemical composition in a non-metric multidimensional scaling analysis $(M D S)$. A canonical analysis of principal components $(C A P)$ was also carried out in order to predict group membership and help confirm the effectiveness of the functions in discriminating among the groups.

All behavioral data were analyzed using generalized linear mixed models (GLMM), which included colony-of-origin as a random factor, except for At. Cephalotes, for which only one colony was used and the data, therefore, analyzed with a generalized linear model (GLM). For colony-level assays, time point nested within colony was used as a random factor. For colony-level assays, number of ants and number of MORs were analyzed using a Poisson distribution and a log-link function. All individual-level MOR experiments were compared using binomial distributions and a logit-link function, 
with stimuli in the first experiment, and stimuli and caste or age in the second experiment, as factors. Non-significant interaction terms were removed stepwise in all cases to obtain the minimum adequate models. Sequential Bonferonni corrections were used to adjust for multiple comparisons during pairwise post-hoc testing. All behavioral analyses were performed in SPSS (v.20 SPSS Inc., Chicago, IL, USA).

\section{Results}

Chemical Composition Alarm pheromone composition differed among the seven species tested $\left(\mathrm{F}_{6,27}=20.3\right.$; $P<0.001)$. The species differed in their compositions, except for Ac. octopsinosus and Ac. echinatior $(P=0.521)$, and also At.cephalotes and $S$. amabilis $(P=0.604)$. There was little congruence across genera between their phylogeny and the chemical similarity of their alarm pheromone (Fig. 1a). This was supported by MDS analysis, which separated the species into three clusters, with the Acromyrmex species clustering together mostly differentiated from other attines by the presence of 3-octanone (Fig. 1b). Interestingly, S. amabilis clustered with the two Atta species, differentiated from the other attines by the presence of 4-methyl-3-heptanone. Trachymyrmex cornetzi and Ap. pilosum made up the third cluster and were both mostly distinguished from the other two clusters by the almost complete lack of either 4-methyl-3-heptanone or 3-octanone. In total, 29 compounds of high volatility occurred consistently in one or more of the species. Of these, 24 compounds could be identified with a high degree of confidence, including all of the most abundant peaks (Table 1). The CAP analysis correctly identified species in $91 \%$ of the 34 samples. Misclassification of species only occurred in 3 out of the 7 species, with $25 \%$ of samples being misclassified for At. cephalotes (1/4 samples), At. colombica (1/4) and S. amabilis (1/4), in all cases as other species within this cluster.

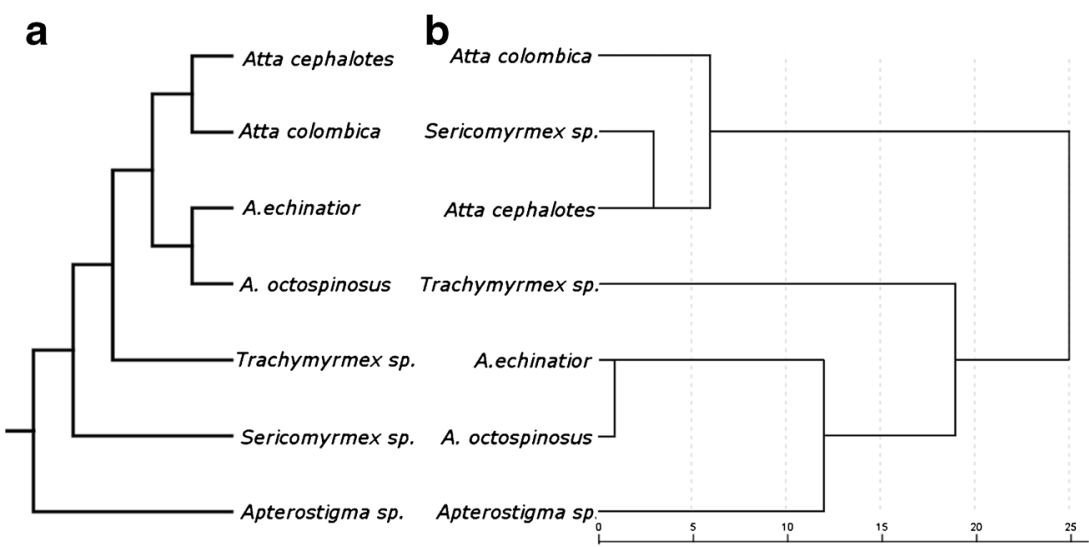

C

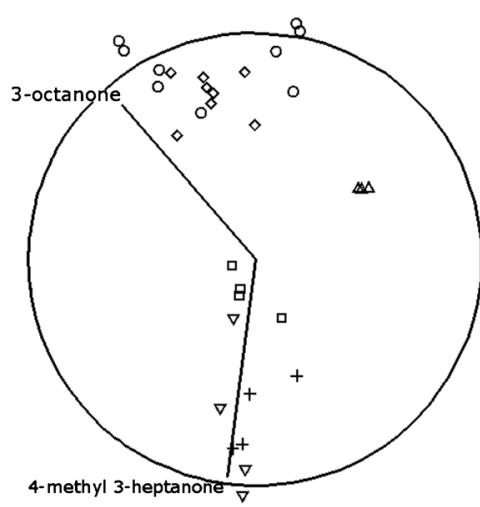

Fig. 1 Composition of the alarm pheromone of seven attine fungusgrowing ants species. (a) Phylogeny of the seven attine species (adapted from Schultz and Brady 2008) and (b) dendrogram showing similarity in the composition of alarm pheromone composition, calculated using Euclidian distances, with shorter distances between chemical profiles indicating greater similarity. (c) Multi-directional scaling (MDS) plot, showing the similarity of alarm pheromone composition for the seven attine species: Apterostigma pilosum (open upright triangles), Trachymyrmex cornetzi (rotated crosses),
Sericomyrmex amabalis (crosses), Acromyrmex echinatior (open diamonds), Acromyrmex octospinosus (open circles), Atta cephalotes (open upturned triangles) and Atta colombica (open squares) calculated using Euclidian distances. Each symbol is from a sample of 20 workers from one colony, with shorter distances between symbols indicating greater similarity in pheromone composition. Straight lines indicate the main axis of differentiation between the samples and the main chemicals causing this grouping 


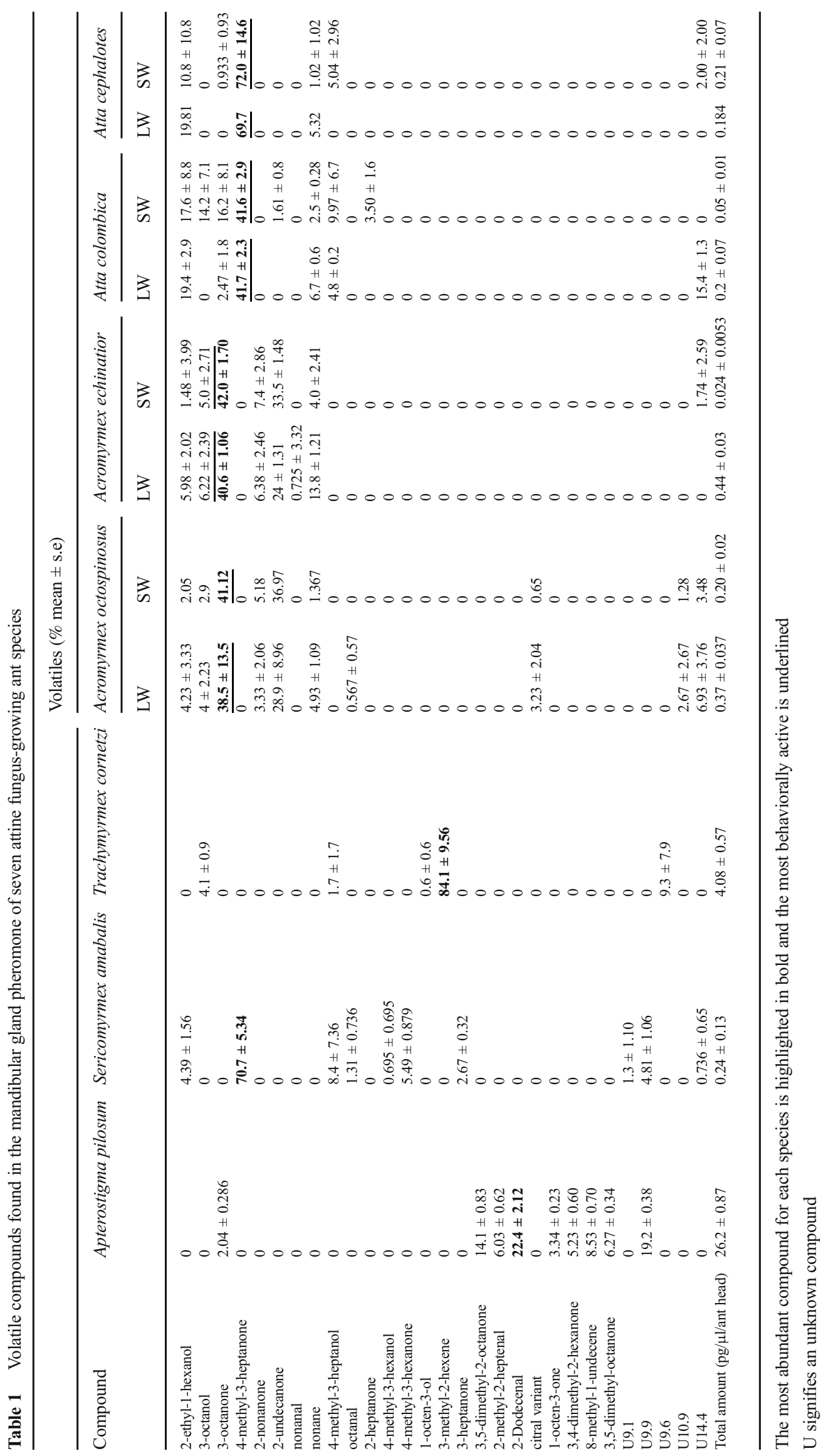


Experiment 1: Behavioral Activity of Compounds in LeafCutting Ants All four species of leaf-cutting ants showed differences in their MOR behavior to the different compounds tested $\left(\mathrm{F}_{8,530}=7.99, P<0.001, \mathrm{~F}_{8,530}=8.93, P<0.001\right.$, $\mathrm{F}_{7,471}=6.62, P<0.001, \chi_{8}^{2}=51.8, P<0.001$ for Ac. echinatior, Ac. octospinosus, At. colombica and At. cephalotes, respectively; Fig. 2). For all species, crushed heads caused more positive MORs than any of the single compounds tested, and there were minimal responses to the water negative control. The most behaviorally active compound for both At. cephalotes and At. colombica was 4-methyl-3-heptanone (Fig. 2). The most behaviorally active compound for Ac. echinatior was 3-octanol, whereas for Ac. octospinosus it was 3-octanone (Fig. 2). There was no difference between large and small workers in their responses to neat compounds for Ac. echinatior, Ac. octospinosus and At. cephalotes $\left(\mathrm{F}_{1,530}=0.204, P=0.653 ; \mathrm{F}_{1,530}=0.315\right.$, $P=0.989, \chi_{1}^{2}=0.995, P=0.319$, respectively), whereas for At. Colombica, small workers were more threat responsive than large workers $\left(\mathrm{F}_{1,471}=19.1, P<0.001\right.$; Fig. $\left.2 \mathrm{c}\right)$.
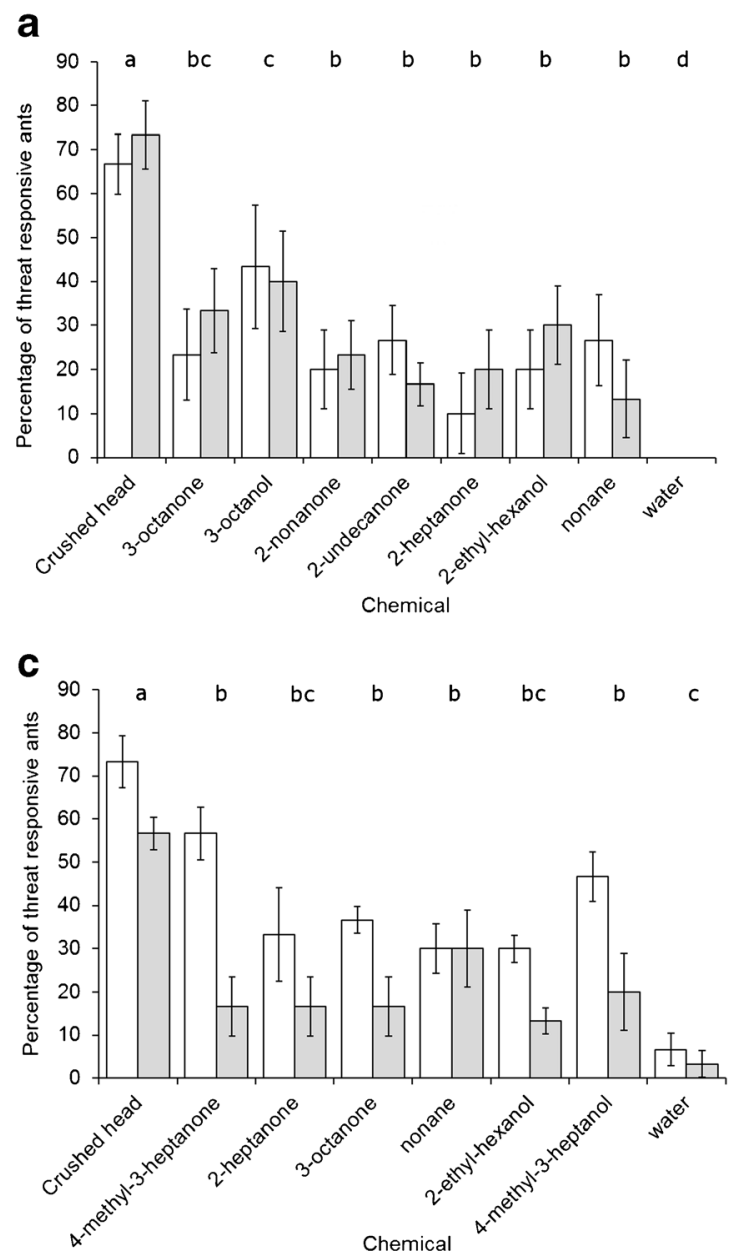

Fig. 2 Behavioral responses of four species of leaf-cutting ants to the most abundant compounds in their alarm pheromones. Mean \pm s.e percentages of ants showing a positive mandible opening response to compounds found in crushed ant heads for 30 small workers (white bars) and 30 large workers (grey bars), sampled evenly among colonies
Experiment 2: the Effect of Caste and Age on Alarm Response in Acromyrmex Ants in all assays responded most strongly to crushed head positive controls, followed by headrealistic doses of the most behaviorally active compound, and showed the least response to the negative control (Fig. 3). For both Ac. echinatior and Ac. Octospinosus, there was no effect of caste on the proportion of ants showing a MOR $\left(\mathrm{F}_{2,265}=0.019, P=0.981, \mathrm{~F}_{2,265}=1.81, P=0.166\right.$, respectively; Fig. 3a,b). However, there was an effect of putative age class on the MOR for both Ac. echinatior and Ac. octospinosus $\left(\mathrm{F}_{2,265}=17.0, P<0.001, \mathrm{~F}_{2,265}=14.0\right.$, $P<0.001$, respectively). Older workers were more threat responsive than younger workers, for all stimuli and for both species (Fig. 3c,d). All color classes, indicating the putative age classes, were different from one another in cuticular color $\left(\mathrm{F}_{2,174}=347.4, P<0.001 ;\right.$ Fig. S2 $)$.

Experiment 3: Colony-Level Assay There were differences among treatments in the numbers of ants within $50 \mathrm{~mm}$ of the

b
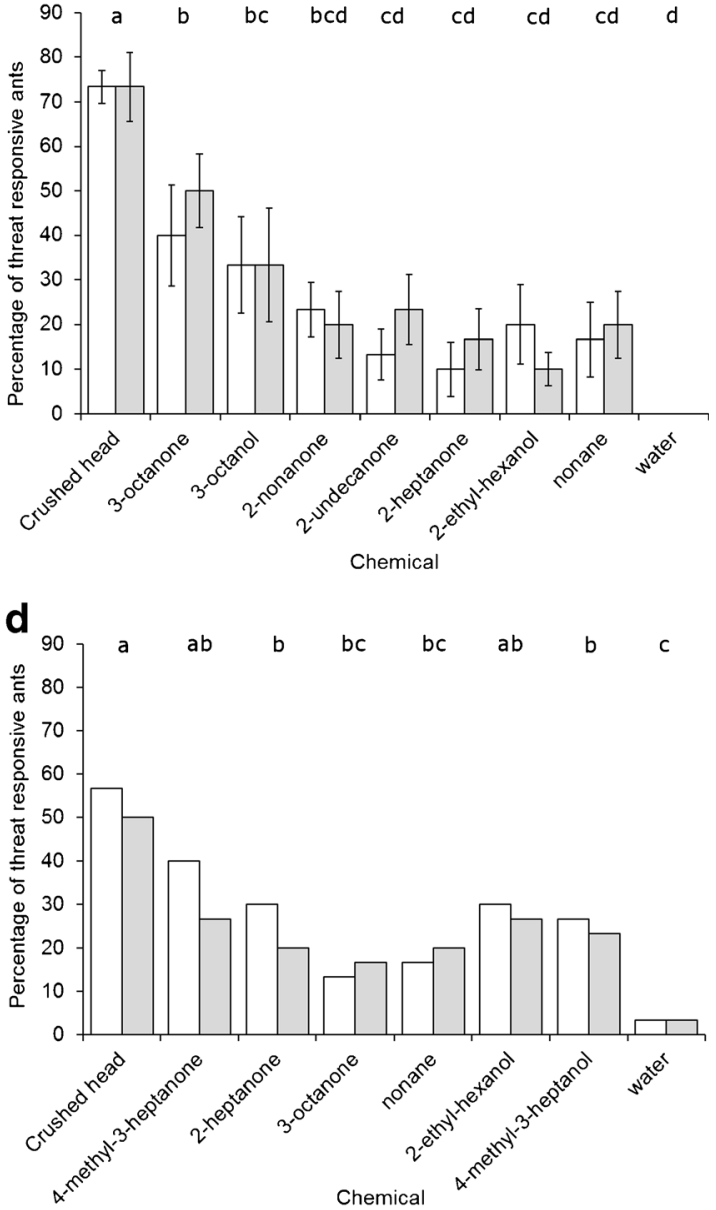

for (a) Acromyrmex echinatior (5 colonies), (b) Acromyrmex octospinosus (5 colonies), (c) Atta colombica (5 colonies) and (d) Atta cephalotes (1 colony). Different letters above columns indicate treatments that differed from each other at $P<0.05$ in pairwise comparisons 
Fig. 3 The effect of caste and age on the behavioral responses of two Acromyrmex leaf-cutting ant species to head-realistic concentrations of the most behaviorally active compounds in their alarm pheromone.

Mean \pm s.e percentages of ants showing a positive mandible opening response (MOR) to solvent negative control (light grey bars), head realistic doses of the most behaviorally active compound (white bars) and crushed nestmate head as a positive control (dark grey bars). Data are for small workers (SW), medium workers (MW) and large workers (LW) of (a) Acromyrmex echinatior, and (b) Acromyrmex octospinosus, and for young (Y), medium $(\mathrm{M})$ and old $(\mathrm{O})$ workers of (c) Acromyrmex echinatior and (d) Acromyrmex octospinosus. Different letters above sets of columns indicate differences among castes or ages of ants at $P<0.05$ in pairwise comparisons a

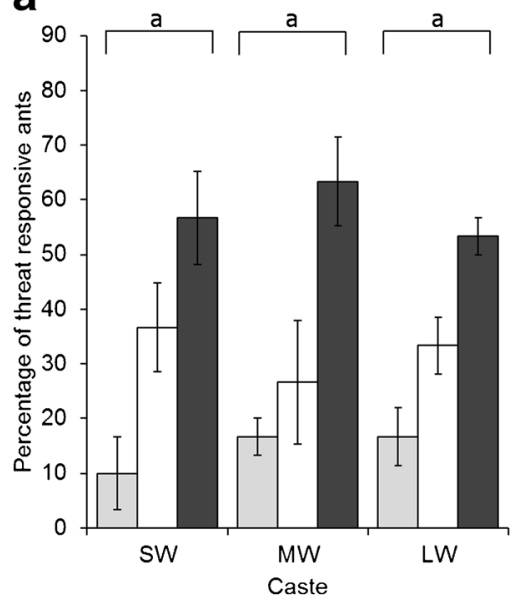

C

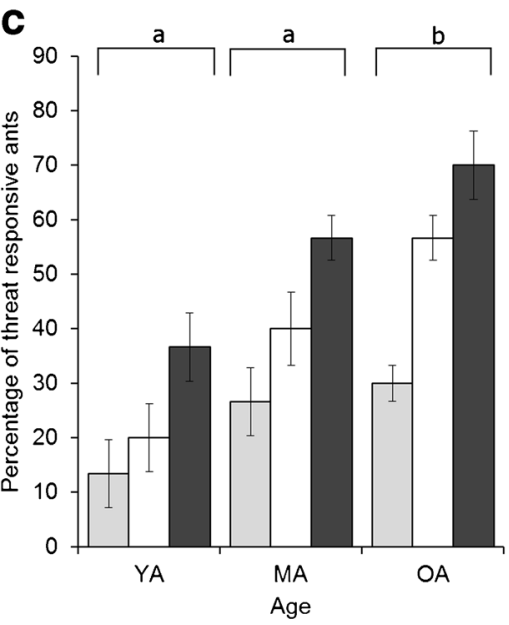

b

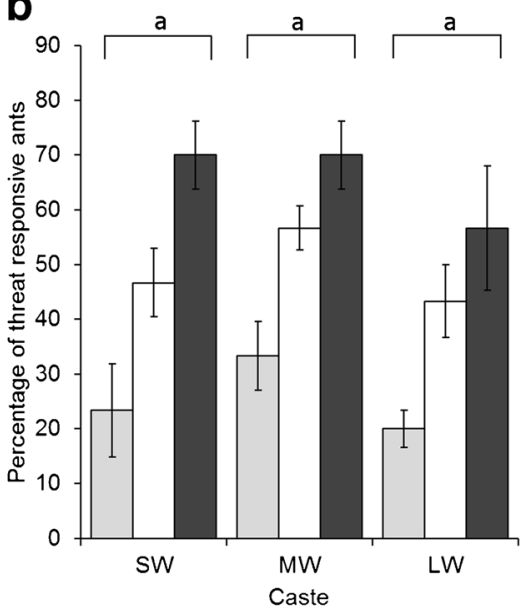

d

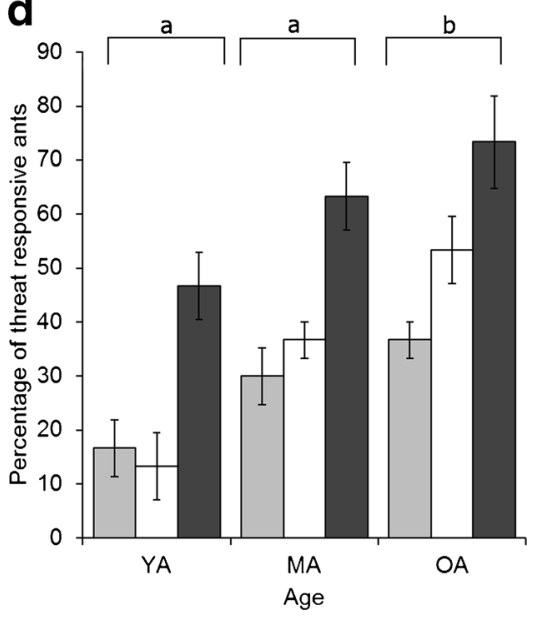

stimulus $\left(\mathrm{F}_{7,136}=18.6, P<0.001, \mathrm{~F}_{7,184}=16.8, P<0.001\right.$ for Ac. echinatior and Ac. octospinosus, respectively). The numbers of ants attracted or arrested near the stimulus were higher in response to crushed heads and either 3-octanol (for $A c$. octospinosus) or 3-octanone (for Ac. echinatior), than in response to the other compounds or the control (Fig. 4a,c). The treatments similarly differed in the numbers of ants exhibiting gaping mandibles following presentation of the stimuli $\left(\mathrm{F}_{7,136}=23.0, P<0.001, \mathrm{~F}_{7,184}=54.2, P<0.001\right.$ for $A c$. echinatior and Ac. octospinosus, respectively), with more ants showing gaping mandibles in response to crushed heads and 3-octanol, or 3-octanone (depending on species), than to the other compounds or control (Fig. 4).

\section{Experiment 4: Confirmation of Behavioral Responses to} Volatiles Released by Ants For both Ac. octospinosus and Ac. echinatior, there were differences among treatments $\left(\mathrm{F}_{4,195}=4.147, P=0.003, \mathrm{~F}_{4,232}=9.361, P<0.001\right.$ for $A c$. echinatior and Ac. octospinosus, respectively) in the numbers of ants exhibiting MOR. Ants showed the highest frequencies of MOR in response to alarmed nestmates with more gaping of mandibles in response to 1 or 8 nestmates than to either a dead nestmate or an empty pot. Identical levels of alarm were produced under red light, simulating darkness for focal ants (Fig. S1).

\section{Discussion}

Contrary to the prediction of little interspecific variation in alarm pheromone composition, we found composition differed across the attine clade, with 4-methyl-3-heptanone being the most abundant compound in S. amabalis and Atta spp., but Acromyrmex spp. utilize different behaviorally active compounds. Furthermore, in contrast to previous studies on Atta, we found that Acromyrmex workers show no caste differences in behavioral response to alarm pheromone, but do exhibit behavioral differences based on putative age class. As in previous studies (e.g., Cammaerts et al. 1983; Moser et al. 1968), no individual compound elicited the same level of behavioral response as the natural pheromone, with the most active compound for each species eliciting a response $60-90 \%$ of that of the natural pheromone, suggesting multiple compounds are needed to elicit full alarm responses.

Although there is a general prediction of a lack of interspecific specificity in alarm pheromones and cues across the 
Fig. 4 Behavioral responses of Acromyrmex octospinosus (a, b) and Acromyrmex echinatior $(\mathbf{c}, \mathbf{d})$ leaf-cutting ant colonies to the most abundant compounds in their alarm pheromones. Mean \pm s.e. number of ants within $50 \mathrm{~mm}$ of the alarm stimulus (a, c) and number of ants exhibiting a mandible opening response (MOR) (b, d). Different letters above columns indicate treatments that differed from each other at $P<0.05$ in pairwise comparisons
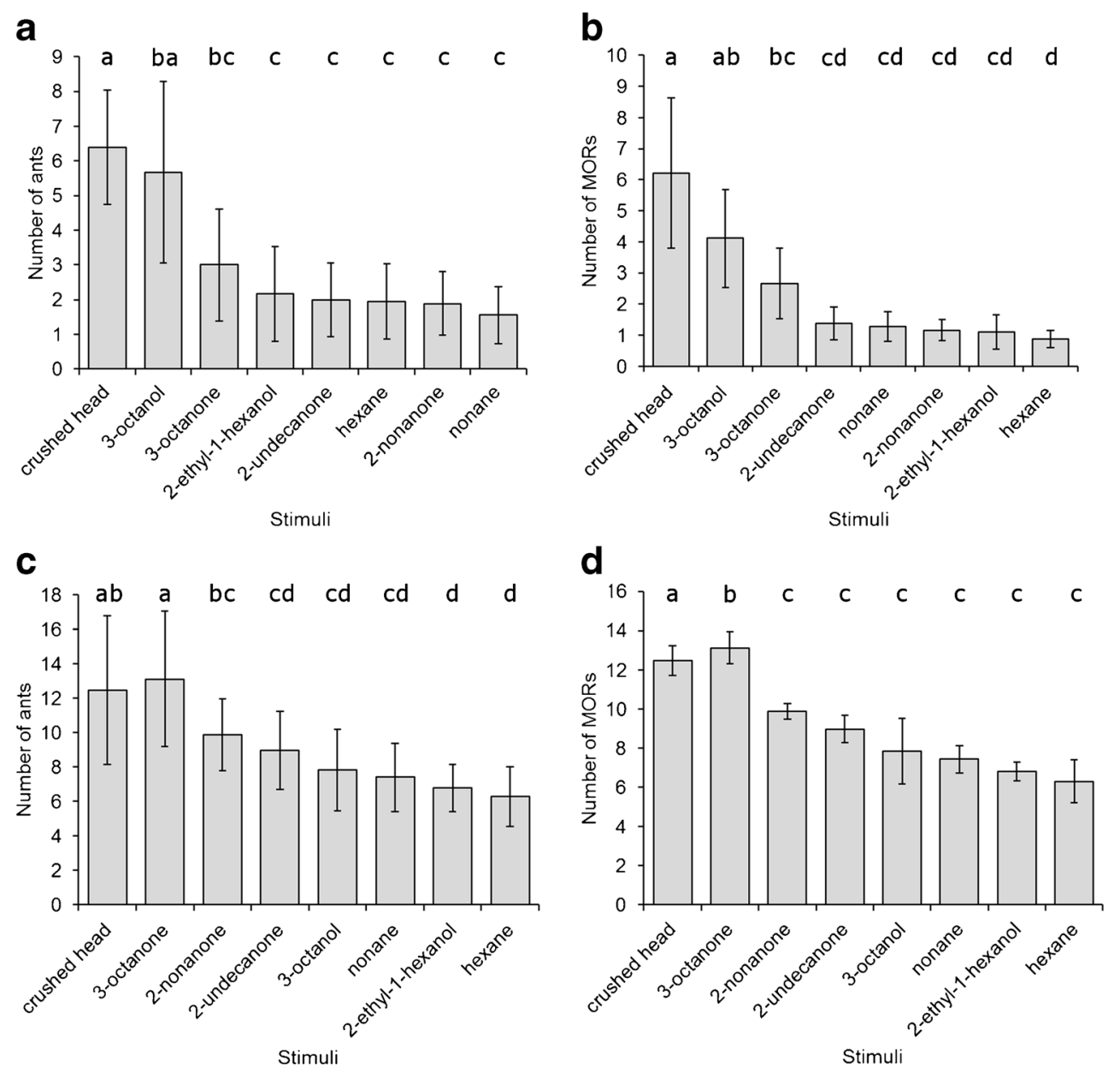

animal kingdom (Blum 1969; Commens and Mathis 1999; Laforsch et al. 2006; Vander Meer and Alonso 1998), it might be expected that taxa with larger, more complex societies might evolve 'better', more informationally rich, mixtures of alarm pheromone (Hölldobler 1995; Wilson and Regnier 1971). This could occur because of the greater quantity of resources they have to defend, and the greater availability and specialization of workers they have with which to respond (Hölldobler and Wilson 1990). The dramatic increase in colony size and complexity across the attines, from tens of monomorphic workers in basal taxa to millions of polymorphic workers in Atta (Weber 1972) suggest potential changes in alarm pheromone composition. However, our results provided no support for either factor affecting pheromone composition. The composition of the mandibular gland pheromone differed little in complexity across the attines and, while the main compound in both Atta leaf-cutting species was 4-methyl-3heptanone in keeping with previous studies of Atta (Francelino et al. 2006; Hernandez et al. 1999; Hughes et al. 2001a; Moser et al. 1968), this compound was also the most abundant in S. amabalis. Furthermore, the other leaf-cutting ant genus Acromyrmex lacked this compound and appears to use 3-octanone or 3-octanol instead. Trachymyrmex cornetzi and Ap. pilosum had unique main constituents compared to the other attines and, although we found very small traces of 4-methyl-3-heptanone, 3-octanol and 3-octanone, previously found in T. septentrionalis and T. seminole (Crewe and Blum 1972), the most abundant peaks for these two attines were 3methyl-2-hexene and 2-dodecenal. One potential explanation for these interspecific differences may be that the components of the mandibular gland secretions serve other purposes, such as being antimicrobial (North et al. 1997). Alternatively, they may have evolved to allow some interspecific recognition, as Acromyrmex and Atta are sympatric competitors, or may be epiphenomenal to differences in diet. The case here is particularly interesting within the genus Acromyrmex, as compounds are found in the same abundance between these sympatric species but have different behavioral activity (3-octanone and 3-ocatanol being most behaviorally active, respectively, for Ac. octospinosus and Ac. echinatior). Regardless of the reason, it seems that there can be remarkable interspecific variability in the composition of alarm pheromone even between closely related taxa, and further investigation of both the proximate and ultimate explanations for this would be worthwhile. 
There was polyethism in the propensity of Acromyrmex workers to respond to alarm pheromone. For both $A c$. echinatior, and Ac. octospinosus, there was no difference in MORs (indicating a focal ant has perceived a threat) among morphological castes (small, medium and large age-matched workers), but there were differences among size-matched young, medium and old putative age classes. Differences in alarm behavior between morphological castes are known in Atta leaf-cutting ants, both in the production of alarm pheromone and behavioral responses to it (Francelino et al. 2006; Hernandez et al. 1999; Hughes et al. 2001a). Smaller workers in Atta respond in higher frequency to conspecific intruders and are more responsive to alarm pheromone (Hughes and Goulson 2001; Wilson 1980; Whitehouse and Jaffe 1996), consistent with our behavioral data. Acromyrmex leaf-cutting ants share many similarities with Atta, including worker polymorphism that affects division of labor, so the lack of an effect of size on response to alarm pheromone in Acromyrmex is somewhat surprising. Previous MOR studies with Acromyrmex have similarly found no difference among physical castes in threat responsiveness (Norman et al. 2014). It may be that the lack of morphological caste differences with respect to nest defence is indicative of Acromyrmex having a simpler division of labour than Atta, or maybe because individuals express a far less extreme alarm response.

Age of workers, even more than morphological caste, is an important and widespread basis for division of labor in social insects, including in Acromyrmex leaf-cutting ants (Camargo et al. 2007; Seeley 1982; Waddington and Hughes 2010). In both Acromyrmex species tested here, putative age class affected the propensity of an individual to show an MOR toward chemical stimuli (with older ants responding more frequently). Older Ac. echinatior individuals are also more threat responsive than young individuals to non-nestmates, and similar relationships with older workers being more aggressive or threat responsive have been reported in many other social insects (Norman et al. 2014; Robinson 1987; Santoro et al. 2015; Waddington et al. 2010; van Wilgenburg et al. 2010). This relationship is likely to be beneficial in insect societies because age polyethism results in older workers spending more time outside of the nest, where they are more likely to encounter threats (Moore et al. 1987). It may be that younger ants are less threat responsive because their longer potential lifespan makes them more valuable to the colony than the older workers, which spend more time outside the nest carrying out behaviors more relevant to alarm cues (Camargo et al. 2007).

The surprising level of interspecific differences seen across the attines in this study highlights the need for detailed, comparative work on the alarm pheromones of fungus-growing ants in particular as well as social insects in general. It will be very interesting to discover whether the logical premise of low interspecific variation in alarm pheromone composition generally holds true and, if not, then why not? The simplicity of alarm pheromones and the interspecific variation in composition that they show in this system offer much potential for studying and understanding the proximate and ultimate basis for the production of pheromones.

Acknowledgements We thank the Smithsonian Tropical Research Institute and Allen Herre for facilities in Gamboa, the Autoridad Nacioanl del Ambiente (ANAM) for permission to collect and export the ants, Elizabeth Hill for technical advice, three anonymous reviewers for their constructive comments on the manuscript and the Biotechnology and Biological Sciences Research Council and Syngenta for funding (BB/ J011339/1). All data supporting this study are provided as supplementary information accompanying this paper.

Open Access This article is distributed under the terms of the Creative Commons Attribution 4.0 International License (http:// creativecommons.org/licenses/by/4.0/), which permits unrestricted use, distribution, and reproduction in any medium, provided you give appropriate credit to the original author(s) and the source, provide a link to the Creative Commons license, and indicate if changes were made.

\section{References}

Adams RMM, Jones TH, Jeter AW et al (2012) A comparative study of exocrine gland chemistry in Trachymyrmex and Sericomyrmex fungus-growing ants. Biochem Syst Ecol 40:91-97. doi:10.1016/j. bse.2011.10.011

Anderson M, Gorley R, Clarke K (2008) PERMANOVA+ for PRIMER: Guide to Software and Statistical Methods

Armitage SAO, Boomsma JJ (2010) The effects of age and social interactions on innate immunity in a leaf-cutting ant. J Insect Physiol 56:780-787

Blum MS (1969) Alarm pheromones. Annu Rev Entomol 14:57-80

Blum MS, Padovani F, Amante E (1968) Alkanones and terpenes in the mandibular glands of Atta species (hymenoptera: formicidae). Comp Biochem Physiol 26:291-299. doi:10.1016/0010-406X(68 )90333-2

Bortolotti L, Costa C (2014) Chemical communication in the honey bee society. CRC Press/Taylor Francis, Boca Raton

Camargo RS, Forti LC, Lopes JFS et al (2007) Age polyethism in the leaf-cutting ant Acromyrmex subterraneus brunneus Forel, 1911 (Hym., formicidae). J Appl Entomol 131:139-145. doi:10.1111 j.1439-0418.2006.01129.x

Cammaerts M-C, Evershed RP, Morgan ED (1983) The volatile components of the mandibular gland secretion of workers of the ants Myrmica lobicornis and Myrmica sulcinodis. J Insect Physiol 29: 659-664. doi:10.1016/0022-1910(83)90019-7

Chivers D, Smith R (1998) Chemical alarm signalling in aquatic predatorprey systems: a review and prospectus. Ecoscience 5:338-352

Collins AM, Rinderer TE, Harbo JR, Bolten AB (1982) Colony defense by africanized and european honey bees. Science 218:72-74. doi:10.1126/science.218.4567.72

Commens AM, Mathis A (1999) Alarm pheromones of rainbow darters: responses to skin extracts of conspecifics and congeners. J Fish Biol 55:1359-1362. doi:10.1111/j.1095-8649.1999.tb02081.x

Crewe RM, Blum MS (1972) Alarm pheromones of the Attini: their phylogenetic significance. J Insect Physiol 18:31-42. doi:10.1016 /0022-1910(72)90062-5

Crewe RM, Blum MS, Collingwood CA (1972) Comparative analysis of alarm pheromones in the ant genus Crematogaster. Comp Biochem Physiol 43:703-716. doi:10.1016/0305-0491(72)90155-1 
Do Nascimento RR, Morgan ED, Billen J et al (1993) Variation with caste of the mandibular gland secretion in the leaf-cutting ant Atta sexdens rubropilosa. J Chem Ecol 19:907-918. doi:10.1007/BF00992527

Francelino MR, Mendonça AL, Do Nascimento RR, Sant'ana AEG (2006) The mandibular gland secretions of the leaf-cutting ants Atta sexdens and Atta opaciceps exhibit intercaste and intercolony variations. J Chem Ecol 32:643-656. doi:10.1007/s10886-005-9020-9

Francelino MR, De Lima MA, Do Nascimento RR et al (2008) Polyethism and nestmate recognition in the alarm reaction of Atta leaf-cutting ants. Physiol Entomol 33:37-42. doi:10.1111/j.13653032.2007.00598.x

Giray T, Guzma E, Aron CW et al (2000) Genetic variation in worker temporal polyethism and colony defensiveness in the honey bee, Apis mellifera. Behav Ecol 11:44-55. doi:10.1093/Beheco/11.1.44

Grüter C, Menezes C, Imperatriz-Fonseca VL, Ratnieks FLW (2012) A morphologically specialized soldier caste improves colony defense in a neotropical eusocial bee. Proc Natl Acad Sci U S A 109:11821186. doi:10.1073/pnas.1113398109

Guerrieri FJ, D'Ettorre P (2008) The mandible opening response: quantifying aggression elicited by chemical cues in ants. J Exp Biol 211: 1109-1113

Hernández JV, Caetano FH (1995) Characterization of the mandible and mandibular glands in different castes of the leaf-cutting ant Atta laevigata (F.Smith) (hymenoptera: formicidae) using scanning electron microscopy. Bol Entomol Venez 10:51-56

Hernandez JV, Cabrera A, Jaffe K (1999) Mandibular gland secretion in different castes of the leaf-cutter ant Atta laevigata. J Chem Ecol 25: 2433-2444

Hernández JV, López H, Jaffe K (2002) Nestmate recognition signals of the leaf-cutting ant Atta laevigata. J Insect Physiol 48:287-295

Hölldobler B (1995) The chemistry of social regulation: multicomponent signals in ant societies. Proc Natl Acad Sci U S A 92:19-22. doi:10.1073/pnas.92.1.19

Hölldobler B, Wilson EO (1990) The ants, 1st edn. Springer, Berlin

Hölldobler B, Wilson EO (2010a) The leafcutter ants. W. W, Norton \& Company, New York, London

Hölldobler B, Wilson EO (2010b) The leafcutter ants: civilization by instinct. W. W, Norton \& Company, New York, London

Hughes WOH, Goulson D (2001) Polyethism and the importance of context in the alarm reaction of the grass-cutting ant, Atta capiguara. Behav Ecol Sociobiol 43:503-508. doi:10.1007/s002650100321)

Hughes WOH, Howse PE, Goulson D (2001a) Mandibular gland chemistry of grass-cutting ants: species, caste, and colony variation. J Chem Ecol 27:109-124. doi:10.1023/A:1005624101743

Hughes WOH, Howse PE, Vilela EF, Goulson D (2001b) The response of grass-cutting ants to natural and synthetic versions of their alarm pheromone. Physiol Entomol 26:165-172. doi:10.1046/j.13653032.2001.00230.x

Kerr WE, Blum MS, Pisani JF, Stort AC (1973) Correlation between amounts of 2-heptanone and iso-amyl acetate in honeybees and their aggressive behaviour. J Apic Res 13:173-176

Laforsch C, Beccara L, Tollrian R (2006) Inducible defenses: the relevance of chemical alarm cues in Daphnia. Limnol Oceanogr 51: 1466-1472. doi:10.4319/1o.2006.51.3.1466

le Roux A, Cherry MI, Manser MB (2008) The audience effect in a facultatively social mammal, the yellow mongoose, Cynictis penicillata. Anim Behav 75:943-949. doi:10.1016/j. anbehav.2007.07.014

Moore AJ, Breed MD, Moor MJ (1987) The guard honey bee: ontogeny and behavioural variability of workers performing a specialized task. Anim Behav 35:1159-1167

Moser JC, Brownlee RC, Silverstein R (1968) Alarm pheromones of the ant Atta texana. J Insect Physiol 14:529-535. doi:10.1016/00221910(68)90068-1
Murakami T, Higashi S, Windsor D (2000) Mating frequency, colony size, polyethism and sex ratio in fungus-growing ants (Attini). Behav Ecol Sociobiol 48:276-284. doi:10.1007/s002650000243

Murphy TG (2006) Predator-elicited visual signal: why the turquoisebrowed motmot wag-displays its racketed tail. Behav Ecol 17: 547-553. doi:10.1093/beheco/arj064

Nault L, Phelan P (1984) Alarm pheromones and sociality in pre-social insects. In: Bell WJ, Carde RT (eds) Chemical ecology of insects. Springer, US, pp 237-256

Norman VC, Hoppé M, Hughes WOH (2014) Old and wise but not size: factors affecting threat response behaviour and nestmate recognition in Acromyrmex echinatior leaf-cutting ants. Insect Soc 61:289-296. doi:10.1007/s00040-014-0355-5

North RD, Jackson CW, Howse PE (1997) Evolutionary aspects of antfungus interactions in leaf-cutting ants. Trends Ecol Evol 12:386389. doi:10.1016/S0169-5347(97)87381-8

Regnier FE, Law JH (1968) Insect pheromones. J Lipid Res 9:541-551. doi:10.1016/0040-4020(77)80262-7

Robinson GE (1987) Modulation of alarm pheromone perception in the honey bee: evidence for division of labor based on hormonally regulated response thresholds. J Comp Physiol A 160:613-619. doi:10.1007/BF00611934

Santoro D, Hartley S, Suckling DM, Lester PJ (2015) The stinging response of the common wasp (Vespula vulgaris): plasticity and variation in individual aggressiveness. Insect Soc 62:455-463. doi:10.1007/s00040-015-0424-4

Schultz TR, Brady SG (2008) Major evolutionary transitions in ant agriculture. Proc Natl Acad Sci U S A 105:5435-5440. doi:10.1073 /pnas.0711024105

Seeley TD (1982) Adaptive significance of the age polyethism schedule in honeybee colonies. Behav Ecol Sociobiol 11:287-293

Shah J (2009) Plants under attack: systemic signals in defence. Curr Opin Plant Biol 12:459-464. doi:10.1016/j.pbi.2009.05.011

Shibao H (1998) Social structure and the defensive role of soldiers in a eusocial bamboo aphid, Pseudoregma bambucicola (Homoptera: Aphididae): a test of the defence-optimization hypothesis. Res Popul Ecol (Kyoto) 40:325-333. doi:10.1007/BF02763464

van Wilgenburg E, Clémencet J, Tsutsui ND (2010) Experience influences aggressive behaviour in the argentine ant. Biol Lett 6:152155. doi:10.1098/rsbl.2009.0616

Vander Meer RK, Alonso E (1998) Pheromone directed behaviour in ants. In: Vander Meer RK, Breed M, Winston M, Espelie K (eds) Pheromone communication in social insects. p 159-192

Verheggen FJ, Haubruge E, Mescher MC (2010) Alarm pheromones chemical signaling in response to danger. In: Litwack $\mathrm{G}$ (ed) Vitamins \& Hormones. p 215-239

Waddington SJ, Hughes WOH (2010) Waste management in the leafcutting ant Acromyrmex echinatior: the role of worker size, age and plasticity. Behav Ecol Sociobiol 64:1219-1228

Waddington SJ, Santorelli LA, Ryan FR, Hughes WOH (2010) Genetic polyethism in leaf-cutting ants. Behav Ecol 21:1165-1169. doi:10.1093/beheco/arq128

Weber NA (1972) Gardening ants, the attines. Mem Am Philos Soc 92:1-146

Wetterer J (1995) Forager size and ecology of Acromyrmex coronatus and other leaf-cutting ants in Costa Rica. Oecologia 104:409-415

Whitehouse M, Jaffe K (1996) Ant wars: combat strategies, territory and nest defence in the leaf-cutting ant Atta laevigata. Anim Behav 51: $1207-1217$

Wilson EO (1980) Caste and division of labor in leaf-cutter ants (hymenoptera: formicidae: Atta): I. The overall pattern in A. sexdens. Behav Ecol Sociobiol 7:143-156. doi:10.1007/BF00299520

Wilson EO, Regnier FE (1971) The evolution of the alarm-defense system in the formicine ants. Am Nat 105:279-289

Wyatt TD (2014) Pheromones and animal behavior: chemical signals and signatures, 2nd edn. Cambridge University Press, Cambridge 